Relations industrielles

Industrial Relations

\title{
Right Reverend New Dealer: John A. Ryan. By Francis L. Broderick. The Macmillian Company, New York; Collier-Macmilian Ltd., London. 1963. 290 pages.
}

\section{Gérard Dion}

Volume 18, numéro 3, juillet 1963

URI : https://id.erudit.org/iderudit/1021422ar

DOI : https://doi.org/10.7202/1021422ar

Aller au sommaire du numéro

Éditeur(s)

Département des relations industrielles de l’Université Laval

ISSN

0034-379X (imprimé)

1703-8138 (numérique)

Découvrir la revue

Citer ce compte rendu

Dion, G. (1963). Compte rendu de [Right Reverend New Dealer: John A. Ryan. By Francis L. Broderick. The Macmillian Company, New York; Collier-Macmilian Ltd., London. 1963. 290 pages.] Relations industrielles / Industrial Relations, 18(3), 436-437. https://doi.org/10.7202/1021422ar

Tous droits réservés (C Département des relations industrielles de l’Université Laval, 1963
Ce document est protégé par la loi sur le droit d'auteur. L'utilisation des services d'Érudit (y compris la reproduction) est assujettie à sa politique d'utilisation que vous pouvez consulter en ligne.

https://apropos.erudit.org/fr/usagers/politique-dutilisation/ 
vés, I'Amérique du Nord ne fait que s'éveiller franchement au problème.

La récente ordonnance du Président Kennedy sur le statut des associations d'employés fédéraux, leur conférant des droits équivalents (souf la grève) à celles des employés ordinaires, montre bien le climat de débloquage qui existe de ce côté chez nos voisins du Sud.

Au Canado, le nouveou régime instauré par le gouvernement Diefenbaker en matière de relations entre le pouvoir central et ses employés, quoique loin de satisfaire ces derniers pleinement, constitue tout de même une amorce dans le sens de l'action présidentielle américaine.

Pour ce qui est du Québec, nous attendons toujours des développements en cette matière, et même le Bill 54 retiré à la dernière session n'opportait à peu près rien de nouveau à une situation désuète et confuse au possible.

Même si la publication de Warner ne s'applique qu'à la situation américaine, de par les témoignages, les opinions, les expériences qu'elle nous présente d'une façon objective et non partisane, elle constitue un document précieux pour servir de point de départ à une étude sérieuse de notre propre situation et amorcer les refontes nécessaires de nos lois en ce domaine.

II s'agit ici d'une série d'articles de différents auteurs organisés et présentés en fonction d'un même thème. On n'a pu éviter, dans cette publication, les dangers inhérents aux ouvrages de même nature, c'est-à-dire, d'une part, le manque d'intégration de l'ensemble des sujets troités, et d'autre part les redites et les recoupements inévitables ò tout travail qui est le fruit de contributions diverses.

Il s'agit donc, non pas d'un traité, mais d'un document de travail, et à ce titre, l'ouvrage est excellent.

Après avoir traité, dans un premier chopitre, rédigé par l'éditeur lui-même, du «climat général nécessaire à la coopération dans la fonction publique, le volume, oux chopitres $2,3,4$ et 5 indique quatre points de vue différents du rôle des relotions ouvrières-patronales dans les services de l'Etat, exprimés par des personnes émanant de différents milieux; direction, syndicats, gouvernements, milieu ocadémique.

Puis les parties III, IV et $V$, s'ottachent ò caractériser, à l'aide d'études monographiques, différents systèmes de relations industrielles oux niveaux local (Partie III), d'Etat (State Government, Partie IV) et fédéral (Portie V)

II s'agit là d'une documentation précieuse colligée dans un même recueil et commentée utilement par les différents collaborateurs.

Enfin une bibliogrophie portant à la fois sur le contexte américain et conadien complète l'ouvrage. Sans minimiser le trovail accompli jusqu'à présent sur le sujet au Canada, notamment par le professeur S.J. Frankel de McGill, nous ne pouvons que souhaiter que des études de ce genre se fassent plus nombreuses au Conoda et au Québec tout particulièrement.

\section{Jean-Réal CARDIN}

\section{Right Reverend New Dealer: John A. Ryan.}

By Francis L. Broderick. The Macmilion Company, New York; Collier-Macmilian Ltd., London. 1963. 290 pages.

Comme les écoliers qui ont un don particulier pour donner des sobriquets à leurs professeurs, le fougeux Father Coughlin, trop célèbre champion de l'anti-communisme, de l'onti-sémitisme et de doctrines monétaires apparentées ou Crédit Social durant la crise économique des années trente, était possé maître dans l'art de qualifier ses adversaires. C'est au cours d'une polémique ovec Mgr John A. Ryan, professeur à I'Université cotholique de Woshington et directeur du Département d'action sociale de la N.C.W.C. qu'il le surnomma Right Reverend New Dealer, qualificatif que son biographe a retenu pour en faire le titre de son ouvrage.

Mgr Ryan est un des hommes qui ont le plus influencé la pensée sociale catholique aux Etats-Unis. Pendant plus de quarante ans, il a été sur la brèche. Par son enseignement d'abord au Séminaire de St-Poul et ensuite ò I'Université catholique de Washington, par les livres qu'il a publiés, par les centaines d'articles qu'il a donnés à différentes revues, par ses conférences qu'il a prononcées dans tous les grands centres des Etats-Unis, par l'orientation qu'il a conférée au Département d'action sociale de la N.C.W.C. dont il a été le directeurfondateur, Mgr Ryan a été dans tous les sens du mot un maître. Autour de lui, comme cela arrive pour tous les grands personnages, il s'est crée un mythe. Ce n'est pas vrai qu'il ait inspiré le New Deal de Roosevelt, comme le veut une certaine légende. Cependant, «all in all, Msgr Ryon was more the New Deal's ambossodor to Catholics than a Catholic legate to the New Deal. He helped create the social mood and the social program that the New Deal embraced. But his greatest service was in acclimating that progrom to Catholic Americanism s. 
$C^{\prime}$ est la vie, les enseignements, les combats de cet homme extraordinaire et pittoresque que l'auteur raconte après ovoir exploré tous les documents écrits et largement utilisé les témoignages oroux de ceux qui ont connu intimement Mgr Ryan. Comme son personnage a été mêlé à tous les problèmes sociaux tant au plan de la doctrine que celui des applications pratiques dans l'évolution qui s'est produite aux EtotsUnis depuis le début du siècle, l'auteur est omené dे foire en quelque sorte oussi une histoire du développement de la pensée sociale dans ce pays durant cette période. Rien de plus intéressant que de constoter comment des positions qui, d̀ un moment donné, paraissent avancées deviennent graduellement acceptées. Mgr Ryan disait luimême à so soeur religieuse en 1918, après la publicotion du fomeux «Bishops' Program for Social Reconstruction 》dont il ovait été l'auteur et qui a vraiment situé l'Eglise américaine dons le domaine social: «After I hove been dead some years, and the things that I have stood for have become regarded as quite safe, men who believe in them will not be regarded as unsafe for that reason. But I may be mistaken as to the time that it will take for the change to come, os things are moving very fast now $>$. Mgr Ryan a vécu assez longtemps pour voir sa prophétie réalisée.

A la fin de l'ouvrage, l'auteur porte le jugement suivant sur Mgr Ryan: Ryan had little impact on the universal Church, but in America he was the peerless leader of, progressive social thinkers who wooed the Church from individualistic fear of the state as the ogent of encroaching socialism and won it back to an acceptance of the state as an instrument of the common good. Ryan served both his Church and his nation: his Church by acclimating its traditional theology to the norms of twentieth-century industrial society and to progressive ottitudes toward social policy; his nation by galvanizing Catholic pressure on the modern state to deal effectively with social problems $\$$. Nous avons connu Mgr Ryan; nous avons lu ses ouvrages; nous voyons encore aujourd'hui ses disciples ou travail. Nous croyons que ce jugement reste en-deço de lo vérité quant ou royonnement international de ce prêtre. En effet, quand on songe aujourd'hui c̀ la position stratégique qu'occupent les Etats-Unis dans le monde entier, et au poids du catholicisme américain dans le cotholicisme universel, on n'ose pas même s'imaginer ce qui serait advenu n'eût été le rôle joué par Mgr Ryan pour aiguillonner les catholiques dans le domaine social et politique. On a dit que le New Deal de Roosevelt avait sauvé les Etats-Unis d'une Révolution dont les répercussions ouraient été mondiales. Les audaces de Mgr. Ryan en pleine conformité avec la doctrine sociale de I'Eglise auront contribué à affaiblir un peu le conservatisme des catholiques américoins et à les amener à prendre conscience de leurs responsabilités sur le plan international.

En plus de nous faire connaître un grand personnage dans le catholicisme américain et de nous mettre au courant de la petite histoire avec laquelle se fait la grande histoire, ce livre est un stimulant pour tous ceux qui s'occupent d'action sociale.

Gérard DION

\section{DROITS DE GÉRANCE \\ et CHANGEMENTS TECHNOLOGIQUES}

Présentation (Emile Gosselin). Nature et importance des changements technologiques (Jean-Paul Deschênes). Propriété, responsabilité et droits de gérance (Gérard Dion). Efficacité, science, participation d la gestion et droits de gérance (Roger Chartier). Changements technologiques et négociations collectives (Jean-Réal Cardin). Arbitrabilité des griefs et changements technologiques (Jean-Jacques Gagnon). Négociabilité et arbitrabilité des changements technologiques (Marius Bergeron). Négociation et arbitrage dans le domaine des changements technologiques (Marcel Pépin, W. Gordon Donnelly, Yvan Legault, Jean Sirois). Le syndicalisme ouvrier face aux changements technologiques (Lewis A. Coser).

1 volume, 149 pages - Prix $\$ 3.00$

LES PRESSES DE L'UNIVERSITÉ LAVAL 